\title{
Smoking as Epidemic: Modeling and Simulation Study
}

\author{
Sintayehu Agegnehu Matintu \\ School of Mathematical and Statistical Sciences, Hawassa University, Hawassa, Ethiopia
}

\section{Email address:}

sinabeba@yahoo.com

\section{To cite this article:}

Sintayehu Agegnehu Matintu. Smoking as Epidemic: Modeling and Simulation Study. American Journal of Applied Mathematics. Vol. 5, No. 1, 2017, pp. 31-38. doi: 10.11648/j.ajam.20170501.14

Received: December 4, 2016; Accepted: December 23, 2016; Published: February 23, 2017

\begin{abstract}
In this paper we propose smoking epidemic model which analyzes the spread of smoking in a population. The model consists of five compartments corresponding to five population classes, namely, potential-moderate-heavy-temporarily recoveredpermanently recovered class. The basic reproduction number $R_{0}$ has been derived, and then the dynamical behaviors of both smoking free equilibrium and smoking persistent equilibrium are analyzed by the theory of differential equation, and Numerical simulation has been carried out and the results have confirmed the verification of analytical results. Sensitivity analysis of $R_{0}$ identifies $\beta_{1}$, the transmission coefficient from potential smokers to moderate smokers and $\beta_{2}$, the transmission coefficient from potential smokers to heavy smokers, as the most useful parameters to target for the reduction of $R_{0}$.
\end{abstract}

Keywords: Smoking Model, Reproduction Number, Equilibrium Value, Stability, Sensitivity Analysis, Numerical Simulation

\section{Introduction}

Smoking is a practice in which a substance is burned and the resulting smoke breathed in to be tasted and absorbed into the bloodstream. Most commonly the substance is the dried leaves of the tobacco plant which have been rolled into a small square of rice paper to create a small, round cylinder called a "cigarette" [1]. Epidemiology is concerned with the distribution of diseases in populations and the factors that influence the transmission of diseases [2]. One of an interesting area in epidemiology to study is smoking subject. There are a lot of studies that has been done on the epidemics of smoking [3-12]. In order to understand the dynamics of this disease, like many infectious diseases, mathematical models can be used. Most of these models are compartmental models, with the population being divided into compartments with the assumptions about the nature and time rate of transfer from one compartment to another. The basic concepts in these models are that all people in a community start as healthy. Healthy people may become infected by diseases. But infected people may become healthy again in community.

Castillo-Garsow et al. [3] presented a mathematical model for giving up smoking. In their model the total population is classified into three compartments namely; potential smokers, smokers and quitters (former smokers). Later on, Sharomi and Gumel [4] extended the basic model to account for variability in smoking frequency, by introducing two classes of smokers who temporarily and permanently quit smoking. Zaman [5] derived and analyzed a smoking model taking into account the occasional smokers compartment in the basic model. In [6], Z. Alkhudhari, S. Al-Sheikh and S. Al-Tuwairqi adopted the model developed and studied in [6] and considered the effect of peer pressure on temporary quitters. In 2014 [7], Z. Alkhudhari, S. Al-Sheikh and S. AlTuwairqi introduced a new model by dividing the smokers into two subclasses: occasional smokers and heavy smokers, and the impact of these two subclasses on the existence and stability of equilibrium points. They also studied the effect of occasional smokers on potential smokers, and later in 2015 [8] they studied the effect of heavy smokers on potential smokers.

In this paper, we develop a new model based on the idea given in [6,7 and 8] by considering, basically, the effect of both moderate and heavy smokers on the potential smokers, and the effect of heavy smokers on temporarily quit smokers. Furthermore, it assesses the impact of this peer pressure on the existence and stability of steady state solutions, and the 
result is supported with numerical simulation.

\section{Mathematical Model Formulation}

Let the total population size at time $t$ is denoted by $N(t)$. We divide the total population $N(t)$ into five subclasses. These are described as follows: potential smokers (nonsmokers) $P(t)$ but likely to get infected (start smoking) in future, moderate (light) smokers $O(t)$, heavy (addicted) smokers $S(t)$, smokers who temporary quit smoking $Q_{T}(t)$ and smokers who permanently quit smoking $Q_{P}(t)$ such that $N(t)=P(t)+O(t)+S(t)+Q_{T}(t)+Q_{P}(t)$.

\subsection{Flow of People Among the Compartments}

People will join the potential smoker's compartment $P(t)$ at a constant recruitment rate of $\sigma$. This recruitment is due to the total natural birth rate $\sigma N$. Some of these people will vacate this compartment at a constant death rate of $\mu$, due to the total natural death rate $\mu P$. It is considered that the potential smokers start smoking due to peer influence of moderate and heavy smokers, will vacate the potential compartment with the rates $\beta_{1} P \frac{O}{N}$ and $\beta_{2} P \frac{S}{N}$. Here, $\beta_{1}$ is the contact rate between potential and moderate smokers and $\beta_{2}$ is the contact rate between potential and heavy smokers.

The moderate smokers, $O(t)$ are increased when potential smokers start to smoke, with rates $\beta_{1} P \frac{O}{N}$ and $\beta_{2} P \frac{S}{N}$. Some

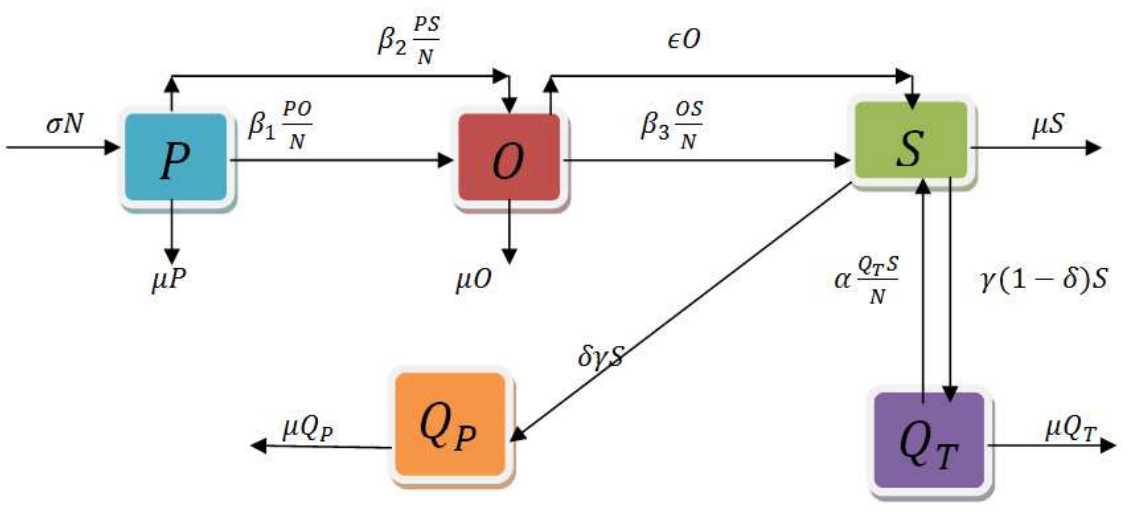

Figure 1. Flow diagram of Smoking Epidemic Model.

\subsection{Model Assumptions}

Here, for the above flow diagram of smoking endemic model, the following assumptions are made.

- The total population is divided into two diseases (smoker) and three non diseases (non smoker) compartments.

- The recruitment rate $(\sigma)$ is different from the natural death rate $(\mu)$ so that the total population is not constant.

- The potential smokers start to smoke under the peer influence of both moderate and heavy smokers.

- All the newly born people are assumed to join only potential smoker's compartment.

- The people of all the compartments will die naturally with the same rate of $\mu$. other people will leave the compartment with the rates $\epsilon O, \beta_{3} O \frac{S}{N}$ and $\mu O$, in which, $\epsilon$ is the rate at which moderate smokers becomes heavy smokers without peer influence and $\beta_{3}$ is the contact rate between moderate and heavy smokers.

People will join into heavy smoker's class $S(t)$ with the rates $\epsilon O, \beta_{3} S \frac{O}{N}$ and $\alpha S \frac{Q_{T}}{N}$. Some others will leave with the rates $\gamma s$ and $\mu S$. Here, $\alpha$ and $\gamma$ are the contact rate between heavy smokes and those who temporarily quit smoking and the rate of quitting smoking, respectively.

People will enter into temporary quitter's compartment $Q_{T}$ with a rate $\gamma(1-\delta) S$ and leave with the rates $\alpha Q_{T} \frac{S}{N}$ and $\mu Q_{T}$, where, $(1-\delta)$ is the fraction of heavy smokers who temporarily quit smoking (at a rate $\gamma$ ).

The permanent quitters, $Q_{P}$ are increased when heavy smokers begin to smoke, with the rate $\gamma \delta S$ and some people of this compartment will die with the rate $\mu Q_{P}$, where, $\delta(0<\delta<1)$ is the remaining fraction of heavy smokers who permanently quit smoking (at a rate $\gamma$ ).

\subsection{Flow Diagram of Smoking Epidemic Model}

Here, we have given the flow diagram of the model. The compartments of the model are represented by rectangular colored boxes. The flow directions of the people among the compartments are represented by directed arrows.

- No other influence to smoke other than smokers.

- Temporarily quitted smokers could only be allowed to relapse into heavy smokers' class.

- Legal age could be considered to start smoking, in the model.

- No person is more popular than another, so that interactions between any two compartments are equally likely.

\subsection{Model Equations}

By adding the rates at which people enter the compartment and subtracting the rates at which people leave the compartment, we get an equation for the rate at which the population of each compartment changes over time. Thus, by considering the above assumptions, smoking 
epidemic model presented in figure 1 may be represented by the following system of first order nonlinear ordinary differential equations:

$$
\begin{gathered}
\frac{d P}{d t}=\sigma N-\beta_{1} P \frac{O}{N}-\beta_{2} P \frac{S}{N}-\mu P, \\
\frac{d O}{d t}=\beta_{1} O \frac{P}{N}+\beta_{2} S \frac{P}{N}-\epsilon O-\beta_{3} O \frac{S}{N}-\mu O,
\end{gathered}
$$

$$
\begin{gathered}
\frac{d S}{d t}=\epsilon O+\beta_{3} S \frac{o}{N}+\alpha S \frac{Q_{T}}{N}-\gamma S-\mu S, \\
\frac{d Q_{T}}{d t}=\gamma(1-\delta) S-\alpha Q_{T} \frac{S}{N}-\mu Q_{T}, \\
\frac{d Q_{P}}{d t}=\gamma \delta S-\mu Q_{P} .
\end{gathered}
$$

With the initial densities

$$
P(0)=P_{0}>0, O(0)=O_{0} \geq 0, S(0)=S_{0} \geq 0, Q_{T}(0)=Q_{T 0} \geq 0, Q_{P}(0)=Q_{T 0} \geq 0 .
$$

By introducing $P=N p, O=N o, S=N s, Q_{T}=N q_{T}$ and $Q_{P}=N q_{P}$, equation (1) can be modified (scaled) as,

$$
\begin{gathered}
\frac{d p}{d t}=\sigma-\beta_{1} p o-\beta_{2} p s-\mu p, \\
\frac{d o}{d t}=\beta_{1} o p+\beta_{2} s p-\epsilon o-\beta_{3} o s-\mu o, \\
\frac{d s}{d t}=\epsilon o+\beta_{3} s o+\alpha s q_{T}-\gamma s-\mu s,
\end{gathered}
$$$$
p(0)=p_{0}>0, o(0)=o_{0} \geq 0, s(0)=s_{0} \geq 0, q_{T}(0)=q_{T 0} \geq 0, q_{P}(0)=q_{T 0} \geq 0 .
$$

$$
\begin{gathered}
\frac{d q_{T}}{d t}=\gamma(1-\delta) s-\alpha q_{T} s-\mu q_{T}, \\
\frac{d q_{P}}{d t}=\gamma \delta s-\mu q_{P},
\end{gathered}
$$

Provided $p+o+s+q_{T}+q_{P}=1$. The initial densities in equation (2) may also be modified as follows:

$\left(d q_{T} / d t\right)=\left(d q_{P} / d t\right)=0$ provided that $o=s=0$. As a result, the smoking free equilibrium point is $E_{0}=$ $(\sigma / \mu, 0,0,0,0)$.

\subsection{The Basic Reproduction Number $\left(R_{0}\right)$}

Like as it has been described in [9], analyzing the differential equations given in equation (3) can also give insight into how smoking spreads and how the spread can be limited. An important tool for analyzing a model of this type is its reproductive number.

The basic reproduction number, denoted $R_{0}$, is defined as the number of new infections produced by a typical infective individual in a susceptible population at a disease free equilibrium [10]. In model (3), taking the infected compartments to be moderate and heavy smokers, the basic reproduction number is then the sum of the transmission rate from potential smokers to moderate smokers divided by the sum of the natural death rate of the moderate smokers population and the proportion of individuals who enter into heavy smoker's compartment, i.e.,

$$
R_{0}=\frac{\beta_{1} o p+\beta_{2} s p}{(\epsilon+\mu) o}
$$

However, the moderate smokers $(o)$ in equation (6) can be computed from equation (3) as follows:

$$
o=\left(\frac{\gamma+\mu}{\epsilon}\right) s
$$

By plugging equation (7) into equation (6), the basic reproduction number at the disease free equilibrium $E_{0}$ is given as,

$$
\mathrm{R}_{0}=\frac{\sigma\left(\beta_{1}(\mu+\gamma)+\epsilon \beta_{2}\right)}{\mu(\epsilon+\mu)(\mu+\gamma)}
$$

As it has been briefly discussed in [10], the dimensionless equilibrium of the differential equations given in equation (3) is obtained by setting $(d p / d t)=(d o / d t)=(d s / d t)=$ 
constant $R_{0}$ tells us that if $R_{0}<1$, then the DFE in $\partial \Omega$, is locally asymptotically stable, and the disease cannot invade the population, but if $R_{0}>1$, then the endemic equilibrium exists in $\Omega^{*}$, and is the DFE is unstable and invasion is always possible.

\subsection{Sensitivity Analysis of $R_{0}$}

It is critical to take various actions to control the system parameters so that $R_{0}$ is remarkably below one. This section is intended to propose some effective measures for achieving this goal. To examine the sensitivity of $\mathrm{R}_{0}$ to each of its parameters, following Arriola and Hyman [13], the normalized forward sensitivity index with respect to each of the parameters is calculated as follows:

$$
\begin{aligned}
& \frac{\partial R_{0} / R_{0}}{\partial \beta_{1} / \beta_{1}}=\frac{\beta_{1}}{R_{0}} \frac{\partial R_{0}}{\partial \beta_{1}}=\beta_{1} \frac{\sigma(\mu+\gamma)}{\mu(\epsilon+\mu)(\mu+\gamma)} \frac{\mu(\epsilon+\mu)(\mu+\gamma)}{\sigma\left(\beta_{1}(\mu+\gamma)+\epsilon \beta_{2}\right)}=\frac{\beta_{1}(\mu+\gamma)}{\beta_{1}(\mu+\gamma)+\epsilon \beta_{2}}>0, \\
& \frac{\partial R_{0} / R_{0}}{\partial \beta_{2} / \beta_{2}}=\frac{\beta_{2}}{R_{0}} \frac{\partial R_{0}}{\partial \beta_{2}}=\frac{\sigma \epsilon \beta_{2}}{\mu(\epsilon+\mu)(\mu+\gamma)} \frac{\mu(\epsilon+\mu)(\mu+\gamma)}{\sigma\left(\beta_{1}(\mu+\gamma)+\epsilon \beta_{2}\right)}=\frac{\epsilon \beta_{2}}{\beta_{1}(\mu+\gamma)+\epsilon \beta_{2}}>0, \\
& \frac{\partial R_{0} / R_{0}}{\partial \sigma / \sigma}=\frac{\sigma}{R_{0}} \frac{\partial R_{0}}{\partial \sigma}=\frac{\sigma\left(\beta_{1}(\mu+\gamma)+\epsilon \beta_{2}\right)}{\mu(\epsilon+\mu)(\mu+\gamma)} \frac{\mu(\epsilon+\mu)(\mu+\gamma)}{\sigma\left(\beta_{1}(\mu+\gamma)+\epsilon \beta_{2}\right)}=1>0 \\
& \frac{\partial R_{0} / R_{0}}{\partial \gamma / \gamma}=\frac{\gamma}{R_{0}} \frac{\partial R_{0}}{\partial \gamma}=\frac{\gamma \beta_{1}(\mu+\gamma)-\gamma\left(\sigma\left(\beta_{1}(\mu+\gamma)+\epsilon \beta_{2}\right)\right)}{\mu(\epsilon+\mu)(\mu+\gamma)^{2}} \frac{\mu(\epsilon+\mu)(\mu+\gamma)}{\sigma\left(\beta_{1}(\mu+\gamma)+\epsilon \beta_{2}\right)}=\frac{-\gamma \sigma \epsilon \beta_{2}}{\sigma(\mu+\gamma)\left(\beta_{1}(\mu+\gamma)+\epsilon \beta_{2}\right)}<0, \\
& \frac{\partial R_{0} / R_{0}}{\partial \gamma / \gamma}=\frac{\mu}{R_{0}} \frac{\partial R_{0}}{\partial \mu}=\frac{\mu}{R_{0}} \frac{\sigma \mu \beta_{1}(\mu+\gamma)(\epsilon+\mu)-((\epsilon+\mu)(\mu+\gamma)+\mu(\mu+\gamma)+\mu(\epsilon+\mu))\left(\sigma\left(\beta_{1}(\mu+\gamma)+\epsilon \beta_{2}\right)\right)}{\mu(\epsilon+\mu)(\mu+\gamma)\left(\sigma\left(\beta_{1}(\mu+\gamma)+\epsilon \beta_{2}\right)\right)} \\
& <\frac{\sigma \mu \beta_{1}-\left(\sigma\left(\beta_{1}(\mu+\gamma)+\epsilon \beta_{2}\right)\right)}{\sigma \mu\left(\beta_{1}(\mu+\gamma)+\epsilon \beta_{2}\right)}=\frac{-\sigma\left(\beta_{1} \gamma+\epsilon \beta_{2}\right)}{\sigma \mu\left(\beta_{1}(\mu+\gamma)+\epsilon \beta_{2}\right)}<0, \\
& \frac{\partial R_{0} / R_{0}}{\partial \epsilon / \epsilon}=\frac{\epsilon}{R_{0}} \frac{\partial R_{0}}{\partial \epsilon}=\frac{\sigma \epsilon\left(\beta_{2} \mu-\beta_{1}(\mu+\gamma)\right)}{(\epsilon+\mu)\left(\sigma\left(\beta_{1}(\mu+\gamma)+\epsilon \beta_{2}\right)\right)}<0, \text { for } \beta_{2} \mu<\beta_{1}(\mu+\gamma),
\end{aligned}
$$

It can be seen that, among these six parameters, $R_{0}$ is the most sensitive to the change in $\beta_{1}, \beta_{2}$ and $\sigma$. An increase or decrease in $\sigma$ will cause an increase or decrease in $R_{0}$ with the same proportion. Physically, however, decreasing the natural birth rate $\sigma$ is neither practical nor ethical. An increase (or decrease) in the value of $\beta_{1}$ or $\beta_{2}$ leads to a corresponding increase (or decrease) in $R_{0}$. As opposed to this, the other two parameters $\mu$ and $\gamma$ have an inversely proportional relationship with $R_{0}$, an increase in $\mu$ and $\gamma$ will bring about a decrease in $R_{0}$, with a proportionally smaller size of decrease. Recall that $\mu$ is the natural death rate of the population. It is clear that increase in $\mu$ is neither ethical nor practical. Additionally, the parameter $\epsilon$ may have directly or inversely proportional relationship with the reproduction number. Thus we choose to focus on one of three parameters: either $\beta_{1}$, the transmission rate from potential smokers to moderate smokers or $\beta_{2}$, the transmission rate from potential smokers to heavy smokers or $\gamma$, the rate of quitting smoking. Given $R_{0}$ 's sensitivity to $\beta_{1}, \beta_{2}$ and $\gamma$, it seems sensible to focus efforts on the reduction of $\beta_{1}$ and $\beta_{2}$. In other words, this sensitivity analysis tells us that prevention is better than quitting smoking.

\subsection{Local Stability of Smoking Free Equilibrium}

The local stability of $E_{0}=(\sigma / \mu, 0,0,0)$ is then determined from the signs of the eigenvalues of the Jacobian matrix. At the smoking free equilibrium $E_{0}$, the Jacobian matrix is given by:

$$
J\left(E_{0}\right)=\left[\begin{array}{c}
-\mu-\frac{\beta_{1} \sigma}{\mu}-\frac{\beta_{2} \sigma}{\mu} 0 \\
0 \frac{\beta_{1} \sigma}{\mu}-\epsilon-\mu \frac{\beta_{2} \sigma}{\mu} 0 \\
0 \epsilon-\gamma-\mu 0 \\
00 \gamma(1-\delta)-\mu
\end{array}\right]
$$

The characteristic equation of this matrix is given by det $\left(\lambda I-J\left(E_{0}\right)\right)=0$, where $I$ is a square identity matrix of order 4 . The equation thus becomes

$$
(\lambda+\mu)^{2}\left(\lambda^{2}+b_{1} \lambda+b_{2}\right)=0,
$$

Where,

$$
b_{1}=\epsilon+\gamma+2 \mu-\frac{\beta_{1} \sigma}{\mu}, b_{2}=(\mu+\epsilon)(\gamma+\mu)\left(1-R_{0}\right) \text {. }
$$

The roots of the characteristic equation are the eigenvalues of the Jacobian matrix. It is clear that the one eigenvalue is determined from $(\lambda+\mu)^{2}=0$, and is $\lambda_{1}=-\mu$. All the other remaining eigenvalues are determined from the quadratic equation 


$$
\lambda^{2}+b_{1} \lambda+b_{2}=0
$$

Now by using Routh-Hurwitz criterion [15], it can be stated that the smoking free equilibrium $E_{0}$ is locally asymptotically stable if and only if the following inequalities satisfied: $b_{1}>0$ and $b_{2}>0$.

Theorem 1: If $R_{0}<1$, then $b_{2}$ and $b_{1}$ are greater than zero.

Proof: Since each parameter's value is greater than zero, it is trivial that $b_{2}>0$ if $R_{0}<1$. Because $\epsilon \beta_{2}>0, \frac{\sigma\left(\beta_{1}(\mu+\gamma)\right)}{\mu(\epsilon+\mu)(\mu+\gamma)}=\frac{\sigma \beta_{1}}{\mu(\epsilon+\mu)}<\frac{\sigma\left(\beta_{1}(\mu+\gamma)+\epsilon \beta_{2}\right)}{\mu(\epsilon+\mu)(\mu+\gamma)}=R_{0}$. Here, if $R_{0}<1$, then $\frac{\sigma \beta_{1}}{\mu}<(\epsilon+\mu)$. This implies that $b_{1}=\epsilon+$ $\gamma+2 \mu-\frac{\beta_{1} \sigma}{\mu}>(\epsilon+\gamma+2 \mu)-(\epsilon+\mu)=\gamma+\mu>0$.

Hence, all roots of equation (11) have negative real parts if $R_{0}<1$.

So all eigenvalues of the Jacobian matrix are negative if $R_{0}<1$, and hence, $E_{0}$ is locally asymptotically stable. If $R_{0}=1$, then equation (10) may be modified as $(\lambda+$ $\mu)^{2}\left(\lambda^{2}+b_{1} \lambda\right)=0$ which has the roots $\lambda_{1}=-\mu, \lambda_{2}=$ 0 and $\lambda_{3}=\frac{\beta_{1} \sigma}{\mu}-(\epsilon+\gamma+2 \mu)<0$, and hence, $E_{0}$ is locally stable. If $R_{0}>1$, then $b_{2}<0$ and it is clear that the quadratic equation has some positive roots. This leads to conclude that the smoking free equilibrium becomes unstable.

\subsection{Existence and Local Stability of Endemic Equilibrium}

To find the smoking present equilibrium of the system of equation (3) setting $(d p / d t)=(d o / d t)=(d s / d t)=$ $\left(d q_{T} / d t\right)=\left(d q_{P} / d t\right)=0$ provided that at least one of the infected compartments' is non zero. Thus, the model has smoking present equilibrium $E^{*}=\left(p^{*}, o^{*}, s^{*}, q_{T}{ }^{*}, q_{P}{ }^{*}\right)$, where

$$
\begin{gathered}
q_{P}{ }^{*}=\frac{\gamma \delta s^{*}}{\mu}, \\
q_{T}{ }^{*}=\frac{\gamma(1-\delta) s^{*}}{\alpha s^{*}+\mu}, \\
o^{*}=\frac{\left(\varepsilon+\beta_{3} s^{*}\right)(\gamma+\mu)-\alpha \gamma(1-\delta) s^{*}}{\left(\varepsilon+\beta_{3} s^{*}\right)\left(\alpha s^{*}+\mu\right)}, \\
p^{*}=\frac{\delta\left(\varepsilon+\beta_{3} s^{*}\right)\left(\alpha s^{*}+\mu\right)}{\beta_{1}\left(\left(\varepsilon+\beta_{3} s^{*}\right)(\mu+\gamma)-\alpha \gamma(1-\delta) s^{*}\right)+\left(\beta_{2} s^{*}+\mu\right)\left(\varepsilon+\beta_{3} s^{*}\right)\left(\alpha s^{*}+\mu\right)},
\end{gathered}
$$

and $s^{*}$ satisfies the equation $\beta_{1} o^{*} p^{*}+\beta_{2} s^{*} p^{*}-\epsilon o^{*}-$ $\beta_{3} o^{*} s^{*}-\mu o^{*}=0$. Analytically, however, it is tricky to obtain $s^{*}$ as in terms of parameters, and hence the existence of smoking present equilibrium will be verified with numerical simulation.

For the smoking present equilibrium $E^{*}=$ $\left(p^{*}, o^{*}, s^{*}, q_{T}^{*}\right)$, the Jacobian matrix is given as,

$$
J\left(E^{*}\right)=\left[\begin{array}{cccc}
m_{11} & m_{12} & m_{13} & 0 \\
m_{21} & m_{22} & m_{23} & 0 \\
0 & m_{32} & m_{33} & m_{34} \\
0 & 0 & m_{43} & m_{44}
\end{array}\right]
$$

Where,

$$
m_{11}=-\beta_{1} o^{*}-\beta_{2} s^{*}-\mu, m_{12}=-\beta_{1} p^{*}, m_{13}=
$$

$$
\begin{gathered}
-\beta_{2} p^{*}, m_{21}=\beta_{1} o^{*}+\beta_{2} s^{*}, m_{22}=\beta_{1} p^{*}-\epsilon-\beta_{3} s^{*}- \\
\mu, m_{23}=\beta_{2} p^{*}-\beta_{3} o^{*}, m_{32}=\epsilon+\beta_{3} s^{*}, m_{33}=\beta_{3} o^{*}- \\
\gamma-\mu+\alpha q_{T}{ }^{*}, m_{34}=\alpha s^{*}, m_{43}=\gamma(1-\delta)-\alpha q_{T}{ }^{*} \text { and } \\
m_{44}=-\alpha s^{*}-\mu .
\end{gathered}
$$

The characteristic polynomial for $J\left(E^{*}\right)$ is given by det $\left(\lambda I-J\left(E^{*}\right)\right)=0$, where $I$ is a square identity matrix of order 4 . The polynomial becomes

$$
\lambda^{4}+b \lambda^{3}+c \lambda^{2}+d \lambda+e=0,
$$

Where,

$$
\begin{gathered}
b=-\left(m_{11}+m_{22}+m_{33}+m_{44}+m_{23} m_{32}\right), \\
c=m_{44} m_{33}+m_{23} m_{32} m_{44}+\left(m_{11}+m_{22}\right)\left(m_{44}+m_{33}+\right. \\
\left.m_{23} m_{32}\right)-m_{34} m_{43}+m_{22} m_{11}-m_{12} m_{21}, \\
d=-\left(m_{44} m_{33}+m_{23} m_{32} m_{44}-m_{34} m_{43}\right)\left(m_{22}+m_{11}\right)+ \\
m_{12} m_{21}\left(m_{44}+m_{33}\right)+m_{22} m_{11}\left(m_{44}+m_{33}+m_{23} m_{32}\right)- \\
m_{13} m_{21} m_{32}, \\
e=m_{22} m_{11}\left(m_{44} m_{33}+m_{23} m_{32} m_{44}-m_{34} m_{43}\right)- \\
m_{12} m_{21}\left(m_{44} m_{33}+m_{34} m_{43}\right)+m_{13} m_{21} m_{32} m_{44} .
\end{gathered}
$$

Algebraically, the epidemic equilibrium value is asymptotically stable by Routh-Hurwitz criteria [15], if $b>0, d>0, e>0$ and $b c d>d^{2}+b^{2} e$. These conditions ensure that all of the four eigenvalues found from (13) have negative real parts. However, the stability of the endemic equilibrium $E^{*}$ will be illustrated using numerical simulations, by imposing the value of parameters and initial population of compartments.

\section{Numerical Simulations}

A number of numerical simulations have been carried out using MATLAB to illustrate the dynamics of the system. We use the following initial population such that $p+o+s+$ $q_{T}+q_{P}=1$ :

$$
\begin{aligned}
& \text { 1. }\left(P(0), o(0), s(0), q_{T}(0), q_{P}(0)\right) \\
& \quad=(0.8,0.11,0.075,0.008,0.007)
\end{aligned}
$$

$$
\begin{aligned}
2 .\left(P(0), o(0), s(0), q_{T}(0), q_{P}(0)\right) \\
\quad=(0.75,0.16,0.06,0.02,0.01)
\end{aligned}
$$

$$
\begin{aligned}
& \text { 3. }\left(P(0), o(0), s(0), q_{T}(0), q_{P}(0)\right) \\
& \quad=(0.68,0.18,0.052,0.045,0.043) \\
& \begin{array}{c}
\text { 4. }\left(P(0), o(0), s(0), q_{T}(0), q_{P}(0)\right) \\
\quad=(0.62,0.25,0.05,0.05,0.03)
\end{array}
\end{aligned}
$$

It can be concluded that the model given in this paper can represent the dynamics of Tobacco use in real life if it is possible to find the reasonable values for parameters. The values of some parameters have been studied in some literatures while others don't. Here, we consider the idea presented in $[11,12]$. When a person first becomes a smoker it is not likely that she/he quits for several years since tobacco contains nicotine, which is shown to be an addictive 
drug. We assume $1 / \gamma$ to be the average value of 15 and 25 years, that is, 20 years. Hence, the value of $1 / \gamma$ is set to 7300 days. The natural death rate $\mu$ is per 1000 per year (currently 8 in the US). There is strong evidence that the attitude towards smoking is starting from high (junior) school time. Therefore $1 / \sigma$ is set to be 1095 days.

For the following numerical simulations we use the following parameters: $\sigma=0.0091, \mu=0.0031, \gamma=$ $0.0013, \beta_{1}=0.0085, \beta_{2}=0.0055, \beta_{3}=0.05, \alpha=$ $0.008, \varepsilon=0.005, \delta=0.35$ for $R_{0}<1$, and $\beta_{1}=$ $0.085, \beta_{2}=0.055, \varepsilon=0.05$ and the others are as it is for $R_{0}>1$.

Figure 2 show that every compartment approaches its smoking free equilibrium value $E_{0}$ as time increases. The results show that the smoking free equilibrium value of the system is asymptotically stable, which is as expected from
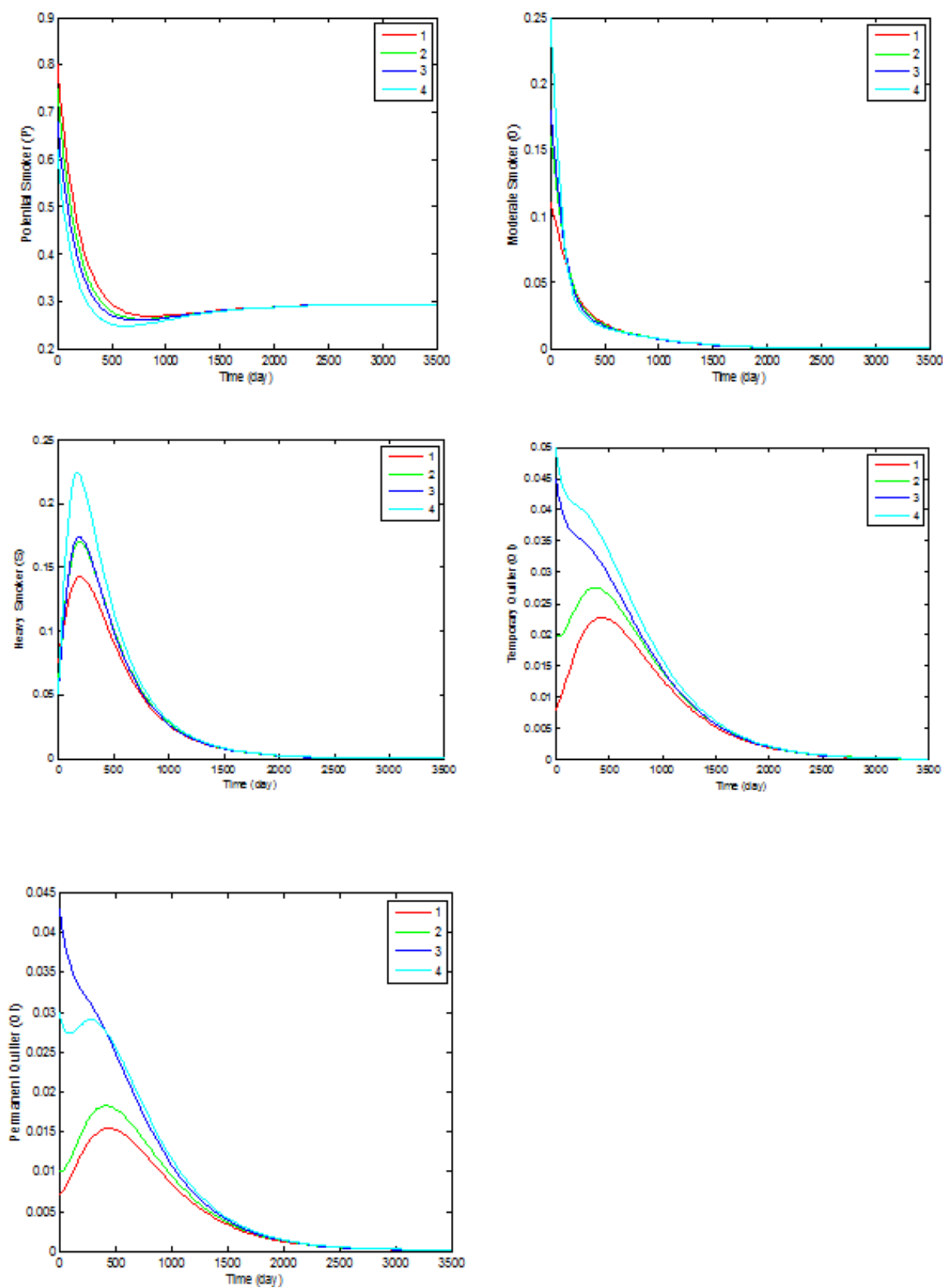

Figure 2. Time series plots of Potential smokers, Moderate smokers, Heavy smokers, Temporary quitter smokers and Permanent quitter smokers with different initial densities for $R_{0}=0.5345<1$.

our theoretical analysis as $R_{0}=\frac{\sigma\left(\beta_{1}(\mu+\gamma)+\epsilon \beta_{2}\right)}{\mu(\epsilon+\mu)(\mu+\gamma)}=0.5345<$ 1. Additionally, the stability of smoking free equilibrium value is not affected by the initial values of each compartment as it is presented in figure 2. On the other hand, we have considered another case in which $R_{0}=3.925 \mathrm{~s}>1$ as it has been shown in figure 3 . The existence and stability of smoking present equilibrium value(s) has been simulated in figure 3 even though it is messy to obtain analytically. Here, the stability of smoking present equilibrium value(s) is/are independent of the initial values. Furthermore, the parameters $\beta_{3}$ and $\alpha$ have no significant on the stability of both smoking free and smoking present equilibrium values as long as their stability depend on the reproduction number $R_{0}$.

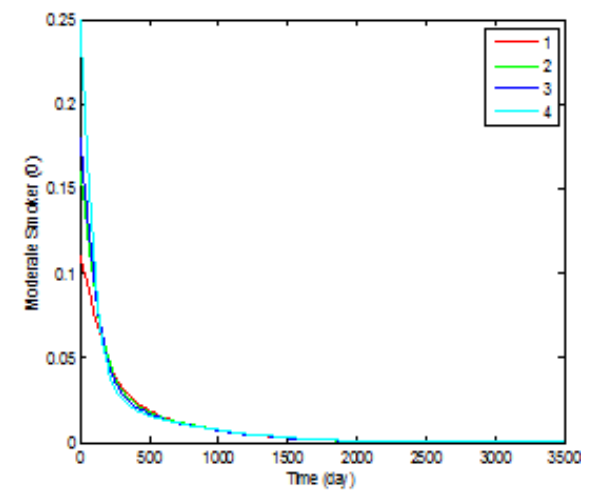



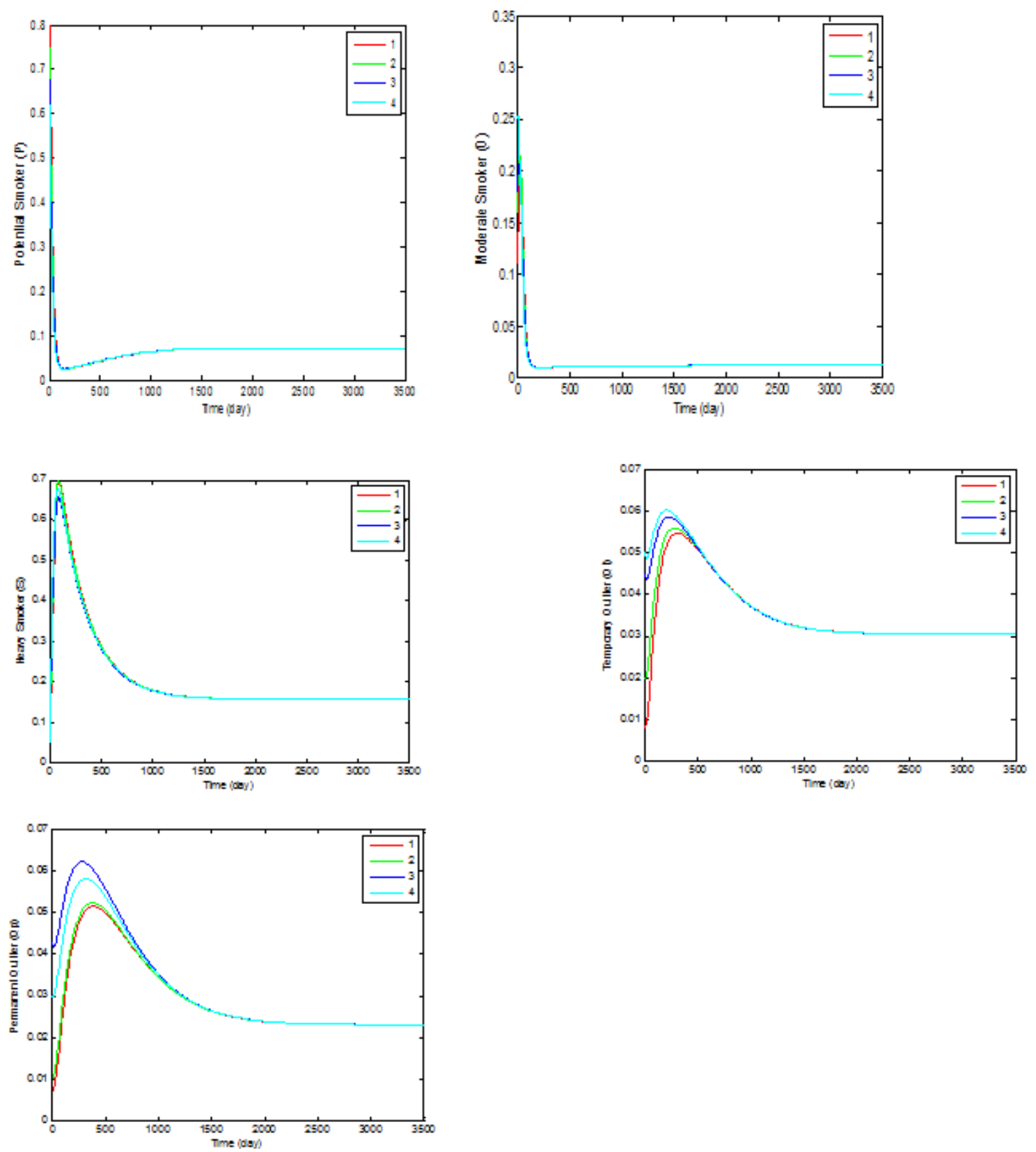

Figure 3. Time series plots of Potential, Moderate, Heavy, Temporary and Permanent quitter smokers with different initial densities for $R_{0}>1$.

\section{Conclusions}

In this paper, we have proposed a mathematical model describing the dynamics of smoking. This model consists of five differential equations describing the rate of change of the subpopulation $P, O, S, Q_{T}$ and $Q_{P}$. The model has smoking free equilibrium and smoking present equilibrium. The threshold $R_{0}$ is derived and it is shown that if $R_{0}<1$, then the smoking will disappear, while if $R_{0}>1$, then the smoking persists with the population which means that after some period of time the smoking will become hazardous. Numerical simulation has been carried out and the results have confirmed the verification of analytical results. Sensitivity analysis of $R_{0}$ identifies $\beta_{1}$, the transmission coefficient from potential smokers to moderate smokers and $\beta_{2}$, the transmission coefficient from potential smokers to heavy smokers, as the most useful parameters to target for the reduction of $R_{0}$. In future work, the possible extensions are to consider that Smoking related death will be introduced in the model and the temporary quitters decide to quit smoking permanently.

\section{References}

[1] http://en.wikipedia.org/wiki/Smoking ( $3^{\text {th }}$ October, 2016)

[2] https://en.wikipedia.org/wiki/Epidemiology (17 $7^{\text {th }}$ November, 2016)

[3] C. Castillo-Garsow, G. Jordan-Salivia, and A. Rodriguez Herrera, Mathematical models for the dynamics of tobacco use, recovery, and relapse, Technical Report Series BU-1505M, Cornell University, Ithaca, NY, USA, 1997. 
[4] O. Sharomi, A. B. Gumel, Curtailing smoking dynamics: A mathematical modeling approach, Applied Mathematics and Computation 195 (2008) 475-499.

[5] G. Zaman, Qualitative behavior of giving up smoking model; Bulletin of the Malaysian Mathematical Sciences Society (2) (2011) 403-415.

[6] Z. Alkhudhari, S. Al-Sheikh, S. Al-Tuwairqi, Global Dynamics of a Mathematical Model on Smoking, ISRN Applied Mathematics vol. (2014), Article ID 847075.

[7] Z. Alkhudhari, S. Al-Sheikh, S. Al-Tuwairqi, The effect of occasional smokers on the dynamics of a smoking model, International Mathematical Forum, 9, No. 25 (2014), 12071222.

[8] Z. Alkhudhari, S. Al-Sheikh, S. Al-Tuwairqi, The effect of heavy smokers on the dynamics of a smoking model, International Journal of Differential Equations and Applications, 14, No. 4 (2015), 1311-2872.

[9] B. Benedict, Modeling Alcoholism as a Contagious Disease: How "Infected" Drinking Buddies Spread Problem Drinking. SIAM News, Vol. 40, No. 3, 2007.
[10] P. van den Driessche, J. Watmough, Reproduction numbers and sub-thershold endemic equalibria for compartmental models of disease transmission, Math. Biosci. 180 (2002) 2948 .

[11] G. Zaman, Y. H. Kang, and I. H. Jung, "Stability analysis and optimal vaccination of an SIR epidemic model," BioSystems, vol. 93, no. 3, pp. 240-249, 2008.

[12] American National Institute of Drug Abuse, "Cigarettes and Other Nicotine Products," http://www.nida.nih.gov/pdf/ infofacts/Nicotine04.pdf.

[13] L. Arriola, J. Hyman, Lecture notes, Forward and adjoint sensitivity analysis: with applications in Dynamical Systems, Linear Algebra and Optimization, Mathematical and Theoretical Biology Institute, Summer, 2005.

[14] Z. Alkhudhari, S. Al-Sheikh, S. Al-Tuwairqi, Global Dynamics of a Mathematical Model on Smoking, ISRN Applied Mathematics vol. (2014), Article ID 847075.

[15] L. Edelstein Keshet, Mathematical Models in Biology. Random House, New York, 1988. SIAM edition. 